\title{
Pyrrole and Pyridine in the Water Environment-Effect of Discrete and Continuum Solvation Models
}

\author{
Krzysztof K. Zborowski* and Jordi Poater*
}

Cite This: ACS Omega 2021, 6, 24693-24699

Read Online

ABSTRACT: Properties of pyrrole and pyridine molecules upon different hydrations were investigated through density functional theory. Complexes of studied molecules with a cluster of 50 water molecules were considered, and the polarizable continuum model of solvation (PCM) was also taken into account. For comparative purposes, all mentioned calculations were repeated for single pyrrole and pyridine molecules and their complexes with one water molecule. Aromaticities of solvated pyrrole and pyridine rings were studied using several geometric- and electronic-based aromaticity criteria. Special attention was paid to studying the properties of formed hydrogen bonds between pyrrole or pyridine and either a single water molecule or several water molecules of the cluster. Overall, a comprehensive description of two very important heterocyclic compounds, that is, pyrrole and pyridine, in both discrete and continuum water solutions, is extensively presented.

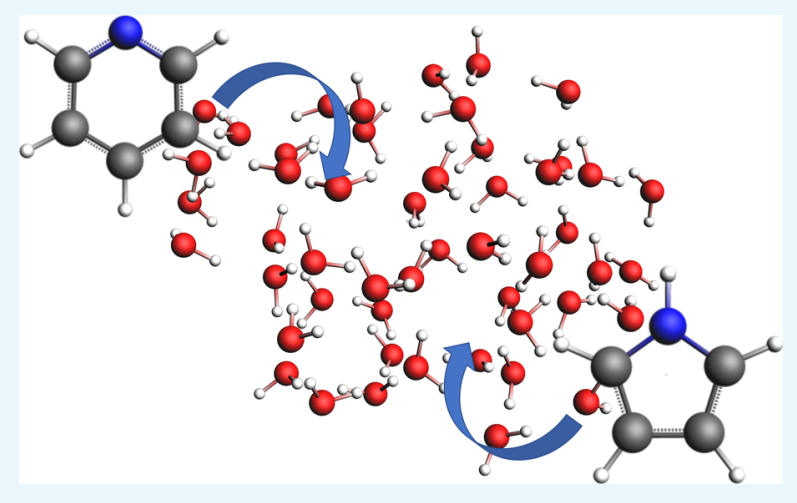

\section{INTRODUCTION}

This project aims to study the influence of the solvent (water) on the properties of pyrrole and pyridine. As an alternative to experimental methods, in silico theoretical approach is applied. Chemical compounds seldom exist as free molecules, that is, not interacting with other molecules. An example of such an interacting system is the so-called solution, where the number of molecules of one compound (solvent) is much larger than that of another compound (solute), which is dispersed among the solvent molecules. The interaction between the solute molecule and the solvent and, consequently, how the solute properties are changed is key to understanding many aspects. Despite this, most of the quantum chemical studies are performed for isolated molecules in the gas phase (GP), mainly due to a much lower computational cost required. However, the improved computing power available makes affordable the study of how the environment (solvent) influences the solute under analysis.

Pyrrole and pyridine are very important heterocyclic compounds. They are the simple models of acidic and basic aromatic systems. For such a reason, theoretical studies of pyrrole and pyridine interaction with water have a long history. Quantum chemical study of the pyrrole-one water and pyridine-one water complexes was previously done. ${ }^{1}$ Molecular structures, vibrational frequencies, dipole moments, and ${ }^{14} \mathrm{~N}$ nuclear quadrupole coupling constants were calculated and compared with some experimental data. The accuracy of density functional theory (DFT) versus MP2 was checked for the pyridine-one water molecule complex. ${ }^{2}$ Methyl-substituted pyridine was also taken into account. ${ }^{3}$ In a more extended study, interactions of pyrrole not only with water but also with HF,
$\mathrm{NH}_{3}, \mathrm{HCl}, \mathrm{H}_{2} \mathrm{~S}$, and $\mathrm{PH}_{3}$ were also a topic of investigation. ${ }^{4} \mathrm{On}$ the other hand, molecular dynamics data of pyrrole $-\mathrm{H}_{2} \mathrm{O}$ and pyrrole- $\left(\mathrm{H}_{2} \mathrm{O}\right)_{2}$ complexes and ground states structures of pyrrole complexes with up to four water molecules were published. ${ }^{5}$ Theoretical studies on pyridine protonation with the presence of one or a few water molecules were published as well. ${ }^{6,7}$ From the above review of previous research, it is clear that studies to date (for simplicity as well as perhaps from the computer limitations of the time) restricted the interactions of pyrrole and pyridine to a limited number of water molecules, most often only one single water molecule. In contrast to the work mentioned above, we have allowed pyrrole and pyridine molecules to interact with multiple water molecules (for details, see the Computational Details chapter). In this way, the description of the behavior of pyrrole and pyridine in the water solution should be a more realistic approach.

The inspiration for this study was performed several years ago through a theoretical investigation of how solvation affects the aromaticity of benzene. ${ }^{8}$ In particular, the noticeable aromaticity of benzene in the GP was proven to persist under a polarizable continuum model of solvation (PCM), as well as with discrete solvation by a cluster of water molecules. More recently, ${ }^{9}$ a study

Received: June 30, 2021

Accepted: July 29, 2021

Published: September 14, 2021 
on the aromaticity of aniline, nitrobenzene, as well as para- and meta-nitroanilines in water was considered. In contrast to benzene, these compounds possess group (s) that can effectively interact with water molecules through the formation of hydrogen bond(s) between solute and solvent molecules. Different models of solvation, from a discrete single water molecule or a continuum solvation model, ${ }^{10-12}$ also with the molecule interacting with either one or two water molecules, and either with or without the continuum model, till solvation by a water cluster were employed. The main aim of that study focused on the substituent effect in solution, despite special emphasis on the effect of solvation on their aromaticity. It was shown how the aromaticity of meta-nitroaniline changed significantly, whereas aromaticity changes of aniline, nitrobenzene, and para-nitroaniline were almost negligible.

The present project aims to go one step further with respect to the above-mentioned research by focusing on the effect of aqueous solvation on two relevant heterocycles, namely, pyrrole and pyridine. Despite both systems being heterocycles with one nitrogen atom, their structures are very different (Figure 1),

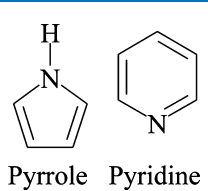

Figure 1. Molecular structures of pyrrole and pyridine.

starting with different ring sizes and hence also their molecular properties, like electronic structures (including aromaticity), ${ }^{13,14}$ stability, ways of interaction with other chemical systems, and also chemical reactivity. Both pyrrole and pyridine can effectively interact with water molecules. However, due to their different chemical structures, pyrrole is a proton donor, while pyridine acts as a proton acceptor. In this way, water itself is also a molecule that can both accept and donate a proton, which might affect the aromatic properties of either pyrrole or pyridine in the aqueous solution. In fact, the relevance of this work relies on the latter.

\section{COMPUTATIONAL DETAILS}

The methodology of this study is the same as that already successfully proven in previous investigations to allow a direct comparison. In a similar way, different models of solvation are taken into account: no solvation (i.e., single pyridine or pyrrole molecules in the GP), single molecule + continuum solvation (PCM), single molecule + water molecule, single molecule + water molecule + PCM, and finally, a pyrrole or pyridine molecule in a water cage. This later cage consists of 50 water molecules, which are freely positioned around the molecule, and then the structure of the whole system is optimized. This number of water molecules has been determined conventionally. We believe that this amount of water is sufficient for simulating the full solvent influence on solute molecule properties, as previously tested. Thus, calculations of energetically optimized geometries for different models of hydration enable us an evaluation of the influence of water solvation on the physicochemical properties of both pyridine and pyrrole. The optimization procedure was repeated 10 times with different starting positions of 50 water molecules for both studied compounds. In this way, 20 different optimized structures, 10 for pyrrole and 10 for pyridine, were obtained.
All DFT calculations presented here were carried out using Gaussian $^{15}$ and GAMESS (version 5 December 2014) ${ }^{16}$ software. We combined the B3LYP functional ${ }^{17}$ and the 6$31 \mathrm{G}^{* *}$ basis set. ${ }^{18,19}$ The $\mathrm{PCM}^{20}$ was applied for the continuum solvation calculations. All geometries were confirmed to be minima by the vibrational frequency analysis. For chemical shielding calculations, the gauge-independent atomic orbital method was used. ${ }^{21}$

The analysis of aromaticity has been carried out by means of different indices of aromaticity with the aim of achieving a reliable trend. In particular, the geometry-based harmonic oscillator model of aromaticity (HOMA), ${ }^{22}$ the magnetic-based nucleus independent chemical shift (NICS), ${ }^{23}$ and the electronic-based aromaticity indices like the para delocalization index (PDI), ${ }^{24}$ fluctuation index (FLU), ${ }^{25}$ and multicenter index (MCI) ${ }^{26,27}$ were employed. HOMA is calculated using bond lengths of the studied ring, and it is equal to 0.0 for the hypothetical Kekule structure of benzene (nonaromatic system reference) and 1.0 for the real benzene structure (aromatic compound reference). NICS is defined as the negative value of the absolute magnetic shielding calculated in the center of the aromatic ring. The more negative the NICS value, the more aromatic the ring. The calculation of the NICS has been limited to the center of the ring because the magnetic shielding out of the plane could depend not only on the aromaticity of the studied ring but also on the proximity of solvent molecules, thus altering the expected trends. Finally, with respect to the electronic-based criteria, PDI is the mean value of the electronic delocalization indices (calculated within the framework of the atoms-in-molecules theory, which are two-center electron sharing measures) for para-related atoms in the rings. PDI index has been defined for six-membered aromatic rings; it has been calculated for pyridine and its water solvated structures. FLU measures the uniformity of the electronic delocalization of a ring with respect to an aromatic bond. Both $I_{\text {ring }}$ and MCI are multicenter indices, with the former only taking into account the Kekule structure, whereas the latter considers all possible arrangements, thus providing a measure of the electron sharing among the atoms considered. Larger values of PDI, $I_{\text {ring, }}$ and MCI and smaller values of FLU stand for the larger aromaticity of that particular ring. ${ }^{28,29}$

The analysis of the electron densities within the AIM framework $^{30}$ (necessary for calculations of electronic indices of aromaticity and charges, as well as for detection and strength evaluation of hydrogen bonds between solvent and solute molecules) was carried out using the AIMAll software ${ }^{31}$ and the ESI-3D program. ${ }^{32,33}$ Additional atomic charge determination was made by the GAPT (generalized atomic polar tensors) ${ }^{34}$ population analysis.

\section{RESULTS AND DISCUSSION}

The influence of the solvent, water in this case, on the selected properties of pyrrole and pyridine is the topic of this study. Interactions of pyrrole and pyridine with an ampholytic solvent like water are very interesting because pyrrole is a model proton donor and pyridine is its model acceptor. In order to achieve this aim, we studied pyrrole and pyridine hydrated in various ways. We started from isolated molecules in the GP (structures labeled as pyrrole-GP and pyridine-GP) that were used as a reference system for the estimation of solvent effects. Then, a single water molecule was added to the pyrrole or pyridine unit (structures pyrrole- $\mathrm{H}_{2} \mathrm{O}$ and pyridine- $\mathrm{H}_{2} \mathrm{O}$ ). With no doubts, for both studied heterocyclic molecules, the strongest interaction 
between a solute molecule and the solvent is the intermolecular hydrogen bond formed, thanks to the presence of the nitrogen atom. However, these bonds are quite different. In the case of pyrrole, the intramolecular hydrogen bond is formed between its $\mathrm{NH}$ group and the oxygen of the water molecule (Figure 2). In

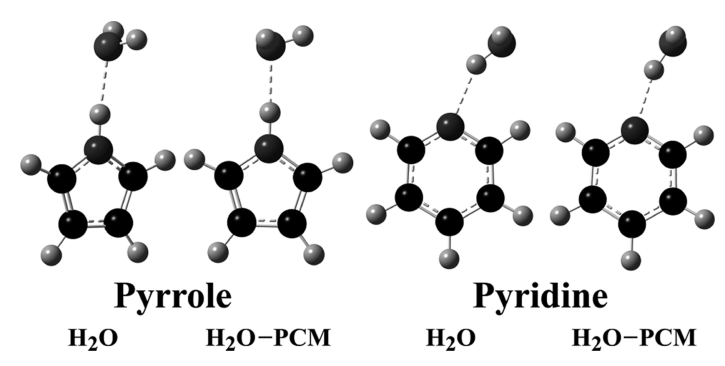

Figure 2. Structures of pyrrole and pyridine with one water molecule.

contrast, in the case of pyridine, such a bond appears between its nitrogen atom and one of the hydrogen atoms of water. Next, we employed a continuum solvation model (PCM) for both systems alone (pyrrole-PCM, pyridine-PCM). Structures in which pyrrole-water or pyridine-water complexes are additionally solvated by the continuum solvation were also studied (pyrrole- $\mathrm{H}_{2} \mathrm{O}-\mathrm{PCM}$, pyridine $-\mathrm{H}_{2} \mathrm{O}-\mathrm{PCM}$ ).

Finally, the above-mentioned model of cluster hydration (a solute molecule surrounded by 50 water molecules, pyrrolecluster and pyridine-cluster) was used. Ten different clusters consisted of pyrrole and 50 water molecules, as well as other ten for pyridine molecule and 50 water molecules were calculated. Representative examples of cluster structures are presented in Figure 3.

Geometries observed in water clusters are usually similar to those of pyrrole- $\mathrm{H}_{2} \mathrm{O}$ and pyridine- $\mathrm{H}_{2} \mathrm{O}$ structures, that is, only $\mathrm{NH} \cdots \mathrm{OH}_{2}$ and $\mathrm{N} \cdots \mathrm{HOH}$ hydrogen bonds are formed for pyrrole and pyridine, respectively (Figure 3 ). Among them, we have nine structures that can be considered as expected for each studied compound. However, we have two very different structures for each of them. In one of the clusters (Figure 3, first from the left), pyrrole interacts with two water molecules instead of one. In this way, a bifurcated hydrogen bond is formed between the $\mathrm{NH}$ of pyrrole and the oxygen of the two water molecules. Nonetheless, the formation of such a hydrogen bond type is quite rare, in only 1 out of the 10 complexes of pyrrole with the 50 water clusters obtained. In the case of pyridine complexes with water clusters, all structures are more similar and form one hydrogen bond with water. However, in one case, pyridine forms a very long hydrogen bond (about 0.7 Å longer) than in other complexes (Figure 3 on the right).

To study the strength of formed intramolecular hydrogen bonds, we investigated their structural and electronic parameters, which are gathered in Table 1 . It is expected that a stronger
Table 1. Bond Lengths (̊̊), Angles (degrees), and Electron Densities at BCPs of Hydrogen Bonds ${ }^{a}$

\begin{tabular}{|c|c|c|c|}
\hline \multicolumn{4}{|c|}{ pyrrole } \\
\hline & $\begin{array}{l}\mathrm{H} \cdots \mathrm{O} \text { bond } \\
\text { length }\end{array}$ & $\mathrm{NH} \cdots \mathrm{O}$ angle & electron density \\
\hline pyrrole- $\mathrm{H}_{2} \mathrm{O}$ & 1.949 & 174.7 & 0.0272 \\
\hline pyrrole- $\mathrm{H}_{2} \mathrm{O}-\mathrm{PCM}$ & 1.890 & 179.1 & 0.0312 \\
\hline $\begin{array}{l}\text { clusters (mean value, } \\
\text { typical structures) }\end{array}$ & 1.966 & 160.0 & 0.0273 \\
\hline $\begin{array}{l}\text { clusters (range, typical } \\
\text { structures) }\end{array}$ & $1.738 / 2.086$ & $135.1 / 177.8$ & $0.0111 / 0.0433$ \\
\hline $\begin{array}{l}\text { clusters (standard } \\
\text { deviation, typical } \\
\text { structures) }\end{array}$ & 0.152 & 13.1 & 0.0105 \\
\hline $\begin{array}{l}\text { clusters (mean value, all } \\
\text { structures) }\end{array}$ & 2.005 & 157.3 & 0.0264 \\
\hline $\begin{array}{l}\text { clusters (range, all } \\
\text { structures) }\end{array}$ & $1.738 / 2.401$ & $130.1 / 177.8$ & $0.0111 / 0.0433$ \\
\hline clusters (standard & 0.195 & 15.4 & 0.0105 \\
\hline \multicolumn{4}{|c|}{ pyridine } \\
\hline & $\begin{array}{l}\mathrm{N} \cdots \mathrm{H} \text { bond } \\
\text { length }\end{array}$ & $\mathrm{N} \cdots \mathrm{HO}$ angle & electron density \\
\hline pyridine- $\mathrm{H}_{2} \mathrm{O}$ & 1.984 & 151.7 & 0.0281 \\
\hline pyridine $-\mathrm{H}_{2} \mathrm{O}-\mathrm{PCM}$ & 1.892 & 162.5 & 0.0346 \\
\hline $\begin{array}{l}\text { clusters (mean value, } \\
\text { typical structures) }\end{array}$ & 1.779 & 165.4 & 0.0449 \\
\hline $\begin{array}{l}\text { clusters (range, typical } \\
\text { structures) }\end{array}$ & $1.730 / 1.873$ & $161.6 / 171.0$ & $0.0353 / 0.0503$ \\
\hline $\begin{array}{l}\text { standard deviation } \\
\text { (typical structures) }\end{array}$ & 0.056 & 3.5 & 0.0060 \\
\hline $\begin{array}{l}\text { clusters (mean value, all } \\
\text { structures) }\end{array}$ & 1.859 & 161.1 & 0.0413 \\
\hline $\begin{array}{l}\text { clusters (range, all } \\
\text { structures) }\end{array}$ & $1.730 / 2.579$ & $122.4 / 171.0$ & $0.0090 / 0.0503$ \\
\hline $\begin{array}{l}\text { clusters (standard } \\
\text { deviation, all } \\
\text { structures) }\end{array}$ & 0.258 & 14.0 & 0.0123 \\
\hline
\end{tabular}

hydrogen bond has a shorter length, an angle closer to $180^{\circ}$, and a larger electron density at the hydrogen bond critical point. Due to the presence of two structures, as mentioned above, that are different from the rest, the mean values, ranges, and standard deviations were calculated (first for expected structures only and then for all calculated structures). It is observed that the mean and standard deviation values (and of course, ranges presented in Table 1 parameters) are only affected if the rarely obtained structures are taken into account. A significant reduction of standard deviation values is observed in the case of the pyridine clusters.

Comparison of the data from Table 1 for pyrrole shows that addition of the continuum solvation to the pyrrole-water

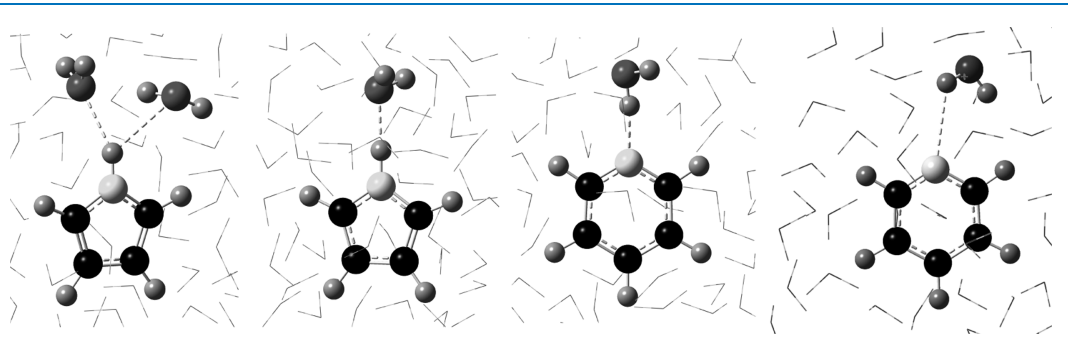

Figure 3. Examples of studied compound complexes with 50 water molecules. 
complex results in the strengthening of all parameters describing the intermolecular hydrogen bond (shorter hydrogen bond length, from 1.949 to $1.890 \AA$, hydrogen bond angle closer to $180^{\circ}$, from 174.7 to $179.1^{\circ}$, and higher electron density at the critical point of the hydrogen bond, from 0.0272 to 0.0312 ). At difference, the interaction of the pyrrole molecule with the cluster of water molecules causes a decrease in the strength of the intermolecular hydrogen bond [longer average value of the hydrogen bond length, $2.005 \AA$, and smaller mean values of hydrogen bond angle, $157.3^{\circ}$, and bond critical point (BCP) electron density, 0.0264]. The exclusion of an unusual structure with a bifurcated hydrogen bond changes these values slightly. In this case, the mean length value is $1.966 \AA$ and the angle is $160^{\circ}$. It is observed how the inclusion of more discrete water molecules in the cluster changes significantly the properties of the hydrogen bond of the pyrrole molecule with water. Thus, the introduction of one water molecule in both $\mathrm{H}_{2} \mathrm{O}$ and $\mathrm{H}_{2} \mathrm{O}-\mathrm{PCM}$ complexes results in the formation of an attractive force between a water molecule and a solute molecule. In contrast, when introduced in a cluster, the water molecules involved in the solute-water hydrogen bonds are attracted also by other water molecules. Such attractive interactions with other water molecules make the pyrrole-water hydrogen bond in the cluster become weaker. However, it should be noted that the ranges of all parameters in complexes with many water molecules are quite wide, and even for some cluster complexes, stronger hydrogen bonds than in pyrrole- $\mathrm{H}_{2} \mathrm{O}$ and pyrrole$\mathrm{H}_{2} \mathrm{O}-\mathbf{P C M}$ structures are also observed. The reason must be found in those few particular cases, that is, only 1 out of 10 systems, as mentioned above, that more largely differ.

Similarly, in the case of pyridine, we also observe the strengthening of the hydrogen bond after including the PCM model. All parameters describing the hydrogen bond strength are shifted toward a stronger hydrogen bond (shorter hydrogen bond length, from 1.984 to $1.892 \AA$, hydrogen bond angle closer to $180^{\circ}$, from 151.7 to $162.5^{\circ}$, and larger electron density at the critical point of the hydrogen bond, from 0.0281 to 0.0346 ). However, at difference with pyrrole, the introduction of pyridine into the water cluster complex also drives to a stronger interaction, in agreement with the continuum model. It can be deducted from the shorter bond length (mean value of $1.859 \AA$ ), larger angle (mean value of $161.1^{\circ}$, very similar to that in PCM), and larger electron density at the hydrogen bond critical point (0.0413). Excluding the structure with an extraordinarily long hydrogen bond further reinforces this picture. The effect of the solute-water hydrogen bond weakening, observed for pyrrole, is not present for pyridine. This is because the pyridine-water hydrogen bond is stronger (has higher values of electron density at the hydrogen bond critical points) than the pyrrole-water one. Thus, the influence of other water molecules on it is relatively weaker.

Thus, it is observed how any model of solvation agrees in making the hydrogen bond stronger in pyridine, whereas in the case of pyrrole, there is certain divergency based on the water cluster complex. A compound that interacts more strongly with solvent molecules should be able to mix with the solvent more easily, that is, dissolve more easily. Thus, a difference in the strength of interaction, pyrrole-water versus pyridine-water, should be reflected in the difference in the solubilities of investigated compounds in water. In fact, pyrrole has limited solubility in water, while pyridine mixes with water without limitation, ${ }^{35}$ thus reaching a good agreement between the experiment and our computed data.
Table 2 encloses the relative energies of the pyrrole and pyridine molecules in different environments. The atoms-in-

Table 2. Relative Energies $[\mathrm{kJ} / \mathrm{mol}]$ of the Pyrrole and Pyridine Units in Different Solvent Environments

\begin{tabular}{lll} 
& \multicolumn{1}{c}{ pyrrole } & pyridine \\
$\mathrm{GP}$ & 0.0 & 0.0 \\
$\mathrm{H}_{2} \mathrm{O}$ & 203.7 & 265.0 \\
$\mathrm{PCM}$ & -15.9 & -12.5 \\
$\mathrm{H}_{2} \mathrm{O}-\mathrm{PCM}$ & 157.0 & 247.4 \\
clusters (mean value, typical structures) & 447.8 & 508.5 \\
clusters (range, typical structures) & $322.4 / 547.4$ & $397.4 / 563.3$ \\
clusters (standard deviation, typical & 75.1 & 56.9 \\
$\quad$ structures) & & \\
cluster (mean value, all structures) & 443.1 & 505.4 \\
cluster (range, all structures) & $322.4 / 547.4$ & $397.4 / 563.3$ \\
clusters (standard deviation, all structures) & 72.4 & 54.5
\end{tabular}

molecules method is able to split the total energy of the whole molecular system into atomic contributions. Then, the energies of pyrrole and pyridine units are calculated as a sum of these atomic contributions. Energies of the GP structures have been chosen as zero levels, the reference ones. In order to calculate the relative energies presented in Table 2, the energy of the GP structure was rejected from the energies of structures of the studied molecules in different modes of solvations. In this way, a positive energy value indicates how the GP structure has lower energy than the free pyrrole/pyridine unit in that particular solvation mode and the other way around if we have a negative energy. The lowest energies of pyridine and pyrrole have been detected in the PCM structures (a pyrrole or pyridine molecule inside the continuum hydration model). In this case, the PCM structures are 15.9 and $12.5 \mathrm{~kJ} / \mathrm{mol}$ lower than the corresponding GP structures of pyrrole or pyridine, respectively. At difference, when 1 explicit water molecule or a cluster of 50 water molecules is included, these energies strongly increase for both pyrrole and pyridine. The largest energy increases are observed for the water clusters (443.1 and $505.4 \mathrm{~kJ} / \mathrm{mol}$ for pyrrole and pyridine, respectively). In addition, all values for pyridine are higher than those for pyrrole. A slight lowering of pyrrole and pyridine energies under the PCM solvation is an effect of the construction of this theoretical approximation. Both pyrrole and pyridine are dipole compounds. Thus, their energies in the continuum solvent with high dielectric constants should be stabilized. The situation changes when solute molecules interact with one or more discrete water molecules. In water complexes, with only one water molecule, pyrrole and pyridine molecules are energetically destabilized. In total, energies of these complexes are lower than the sum of energies of their parts (a solvent and a water molecule), but the energy decreases in such complexes are located in the water molecule. The effect of energetic destabilization of the solute molecules intensifies in water clusters. In particular, solute molecules interact with water cages not only by $\mathrm{N} \cdots \mathrm{HO}$ or $\mathrm{NH} \cdots \mathrm{O}$ hydrogen bonds, but water molecules surround also hydrophobic parts of pyrrole and pyridine.

As with the data in Table 1 , the mean values, parameter ranges, and standard deviations were calculated twice. However, it can be seen, somewhat unsurprisingly, that the exclusion of such an unusual structure among the pyridine and pyrrole clusters does not affect these parameters much. Furthermore, the inclusion/exclusion of these rarer cluster structures has very 
little or no effect on the mean values, parameter ranges, and standard deviations of the data that will be presented in both Tables 3 and 4. Therefore, only calculations for all clusters analyzed are presented in Tables 3 and 4 .

Table 3. Charges (in a.u.) of Pyrrole and Pyridine Units in Various Solvent Environments

\begin{tabular}{lcl} 
& \multicolumn{1}{c}{ AIM } & \multicolumn{1}{c}{ GAPT } \\
& Pyridine & \\
$\mathrm{H}_{2} \mathrm{O}$ & -0.0245 & -0.0103 \\
$\mathrm{H}_{2} \mathrm{O}-\mathrm{PCM}$ & -0.0336 & -0.0356 \\
cluster (mean value, all structures) & 0.0820 & 0.0891 \\
cluster (range, all structures) & $0.0440 / 0.0968$ & $0.0462 / 0.1305$ \\
clusters (standard deviation, all & 0.0142 & 0.0228 \\
$\quad$ structures) & & \\
& Pyrrole & \\
$\mathrm{H}_{2} \mathrm{O}$ & 0.0331 & 0.0234 \\
$\mathrm{H}_{2} \mathrm{O}-\mathrm{PCM}$ & 0.0497 & 0.0497 \\
cluster (mean value, all structures) & 0.0464 & 0.1105 \\
cluster (range, all structures) & $0.0165 / 0.0724$ & $0.0711 / 0.1523$ \\
clusters (standard deviation, all & 0.0173 & 0.0271 \\
$\quad$ structures) & & \\
\hline
\end{tabular}

The charge transfers along the different kinds of hydration from pyrrole or pyridine to the solvent or in the opposite direction are collected in Table 3. Both pyrrole and pyridine in the GP and under continuum solvation have total charges equal to zero. In other phases, charges of pyrrole and pyridine are calculated as a sum of atomic contributions. The negative charge of a solute molecule in the $\mathbf{H}_{2} \mathbf{O}, \mathbf{H}_{2} \mathbf{O}$-PCM, or cluster phases indicates the flow of electrons from the solvent toward the solute molecule, whereas a positive value refers to an electron flow from the solute to the solvent (Table 3).

In general, the charge transfer between the solvent and the solute are small based on the data provided by either AIM or GAPT population analyses. Both analyses predict an electron transfer from pyrrole to the solvent molecules following the order $\mathbf{H}_{2} \mathbf{O}<\mathbf{H}_{2} \mathbf{O}-\mathbf{P C M} \approx$ cluster $(0.03,0.0497$, and 0.0464 a.u., with AIM, respectively). Thus, as expected, the continuum model and the water cluster model cause a larger charge transfer. Unexpectedly, a low charge transfer is observed for the GAPT analysis in the case of water clusters.

In contrast, both AIM and GAPT show a transfer from the solvent to pyridine in the cases of $\mathrm{H}_{2} \mathrm{O}$ and $\mathrm{H}_{2} \mathrm{O}$-PCM systems ( 0.02 and 0.03 a.u., with AIM, respectively). This difference can be justified due to the different typology of the hydrogen bond formed in either pyridine or pyrrole (Figure 2), that is, hydrogen bond donor versus hydrogen bond acceptor. However, and more importantly, when the water cluster is considered, there is also a charge transfer from pyridine to the solvent (about 0.08 a.u.), like for pyrrole.

Calculated values of aromaticity indices for pyrrole and pyridine molecules studied here under different hydration conditions are collected in Table 4 . For comparison, previously ${ }^{8}$ calculated values of HOMA, NICS(0), and PDI values for benzene in GP and water cluster environments are also added. When comparing the aromaticity values, we must take into account that PDI can only be applied to six-membered rings, that is, pyridine, whereas NICS may be affected by the size of the ring, that is, six- versus five-membered rings for pyridine and pyrrole, respectively.

First, as it is observed in Table 4, benzene is highly aromatic in the GP, and this is hardly changed in solution. Only NICS is affected in the water cluster, but this could be due to the coupling between the water molecules and the center of the ring where NICS is measured. ${ }^{36}$ This stability of benzene can be attributed to the fact that it has no group that can strongly interact with water. However, this is not the case for either pyrrole or pyridine, as we have seen above. Will these interactions with solvent molecules be translated into their aromaticity and disturb it? The answer is no as it can be seen how also the aromaticity of both pyrrole and pyridine in the water environment is very stable. Thus, all considered systems are proven to be, based on the different calculated criteria, highly aromatic systems also in solution. HOMA index predicts very high aromaticity of pyridine and benzene in all studied

Table 4. Aromaticity Data for the Pyrrole, Pyridine, and Benzene ${ }^{1}$ Rings in Different Environments

\begin{tabular}{|c|c|c|c|c|c|c|}
\hline & HOMA & $\operatorname{NICS}(0)$ & PDI & FLU & $I_{\text {ring }}$ & MCI \\
\hline \multicolumn{7}{|c|}{ Pyrrole } \\
\hline GP & 0.85 & -15.85 & & 0.0065 & 0.0300 & 0.0432 \\
\hline $\mathrm{H}_{2} \mathrm{O}$ & 0.87 & -15.85 & & 0.0050 & 0.0323 & 0.0467 \\
\hline PCM & 0.86 & -15.85 & & 0.0056 & 0.0307 & 0.0463 \\
\hline $\mathrm{H}_{2} \mathrm{O}-\mathrm{PCM}$ & 0.87 & -15.90 & & 0.0043 & 0.0337 & 0.0493 \\
\hline cluster (mean value, all structures) & 0.87 & -14.46 & & 0.0052 & 0.0304 & 0.0439 \\
\hline cluster (range, all structures) & $0.85 / 0.88$ & $-15.20 /-14.01$ & & $0.0040 / 0.0058$ & $0.0287 / 0.0324$ & $0.0408 / 0.0466$ \\
\hline clusters (standard deviation, all structures) & 0.01 & 0.44 & & 0.0006 & 0.0012 & 0.0019 \\
\hline \multicolumn{7}{|c|}{ Pyridine } \\
\hline GP & 0.99 & -8.14 & 0.1024 & 0.0034 & 0.0463 & 0.0688 \\
\hline $\mathrm{H}_{2} \mathrm{O}$ & 0.99 & -8.24 & 0.1014 & 0.0021 & 0.0452 & 0.0669 \\
\hline PCM & 0.99 & -8.11 & 0.1019 & 0.0030 & 0.0457 & 0.0677 \\
\hline $\mathrm{H}_{2} \mathrm{O}-\mathrm{PCM}$ & 0.99 & -8.20 & 0.1007 & 0.0018 & 0.0446 & 0.0656 \\
\hline cluster (mean value, all structures) & 0.99 & -7.78 & 0.0971 & 0.0017 & 0.0405 & 0.0594 \\
\hline cluster (range, all structures) & $0.988 / 0.996$ & $-7.4517 /-8.1954$ & $0.0955 / 0.0988$ & $0.0013 / 0.0028$ & $0.0396 / 0.0418$ & $0.0579 / 0.0619$ \\
\hline clusters (standard deviation, all structures) & 0.002 & 0.25 & 0.0009 & 0.0004 & 0.0008 & 0.0012 \\
\hline \multicolumn{7}{|c|}{ Benzene $^{1}$} \\
\hline GP & 0.96 & -9.84 & 0.1033 & & & \\
\hline PCM & 0.98 & -9.84 & 0.1035 & & & \\
\hline cluster (range) & $0.97 / 0.95$ & $-8.79 /-8.55$ & $0.0993 / 0.0958$ & & & \\
\hline
\end{tabular}


structures. Pyrrole is a bit less aromatic based on geometric data. NICS values for pyrrole and pyridine also hardly change when solvation is introduced. NICS values in water cages are a bit less negative, which suggests a slight decrease in aromaticity. However, NICS values can be affected in the clusters by water molecules, causing a decrease of aromaticity for both pyrrole and pyridine. The reason could be the same as for benzene. Next, with respect to aromaticity measured by means of electronic criteria, the changes are also small in the order of the third decimal in all cases. First, for pyrrole, all FLU, $I_{\text {ring, }}$ and MCI point out a slight increase of aromaticity with solvation. However, the opposite is observed for pyridine based on $I_{\text {ring }}$ and MCI criteria, which show a slight decrease of aromaticity. These trends can be linked to the different hydrogen bond interaction between both heterocycles too, that is, proton donor versus proton acceptor with discrete water. The small divergency among the aromaticity data can be simply a manifestation of a known effect that different aromaticity indices can describe the aromaticity of the same compound in a slightly different way. ${ }^{37}$ The small effect of solvation, either discrete or continuum, on both systems could have already been expected from the impossibility of drawing a resonance structure for any of them bonded to water, thus avoiding any resonance effect in the $\pi$-system. Nonetheless, some systems in which the formation of hydrogen bond results in larger changes in aromaticity were previously reported. However, for significant aromaticity disturbance, the presence of two strongly interacting solvent or other ligand groups (one group donating proton and the second attracting proton) in the ortho position is required. ${ }^{38}$

\section{CONCLUSIONS}

Benzene is a hydrophobic compound, which does not interact strongly with water molecules. Thanks to that, benzene can easily maintain its high aromaticity in a water solution. ${ }^{8}$ The situation in both investigated compounds, pyrrole and pyridine, is reversed. These compounds can effectively interact with water through $\mathrm{NH}$ (pyrrole) or $\mathrm{N}$ (pyridine) groups. Performed calculations give us an insight into the details of both studied compounds' interactions with water. In the case of their complexes with a cluster of 50 water molecules, they interact strongly with the cluster by one water molecule. We determined the geometric and electronic parameters of formed ligand-water hydrogen bonding. It is shown how pyridine forms a stronger hydrogen bond than pyrrole. As a consequence, pyridine dissolves in water much better than pyrrole. Both molecules are energetically destabilized in their water solutions. Population analyses predict a small (about 0.005-0.1) electron transfer from the solute to the solvent in the studied cluster complexes of a solute molecule and many discrete water molecules. More importantly, the aromaticity of investigated compounds in water solution is only slightly perturbed. Thus, the high resistance of aromatic properties in the presence of water molecules, previously observed for benzene, is confirmed. Future studies on compounds in which aromaticity is more affected by a large discrete representation of solvation are going on in our laboratories.

\section{ASSOCIATED CONTENT}

\section{(s) Supporting Information}

The Supporting Information is available free of charge at https://pubs.acs.org/doi/10.1021/acsomega.1c03437.
Hydrogen bond parameters, relative energies of solute molecules in water clusters, values of aromaticity indices, and cartesian coordinates for studied clusters (PDF)

\section{AUTHOR INFORMATION}

\section{Corresponding Authors}

Krzysztof K. Zborowski - Department of General Chemistry, Faculty of Chemistry, Jagiellonian University, 30-387 Kraków, Poland; Email: zborowsk@chemia.uj.edu.pl

Jordi Poater - Departament de Química Inorgànica i Orgànica and Institut de Química Teòrica i Computacional (IQTCUB), Universitat de Barcelona, 08028 Barcelona, Catalonia, Spain; ICREA, 08010 Barcelona, Spain; 이이.org/0000-00020814-5074; Email: jordi.poater@ub.edu

Complete contact information is available at: https://pubs.acs.org/10.1021/acsomega.1c03437

\section{Notes}

The authors declare no competing financial interest.

\section{ACKNOWLEDGMENTS}

The work has been performed under the Project HPCEUROPA3 (INFRAIA-2016-1-730897) with the support of the EC Research Innovation Action under the H2020 Programme. K.K.Z. acknowledges the support of IQTCUB and the computer resources and technical support provided by the Barcelona Supercomputing Center (BSC-CNS). J.P. thanks the Spanish MINECO (PID2019-106830GB-I00 and MDM2017-0767) and the Generalitat de Catalunya (2017SGR348).

\section{REFERENCES}

(1) Martoprawiro, M. A.; Bacskay, G. B. Quantum chemical studies of the pyrrole-water and pyridine-water complexes. Mol. Phys. 1995, 85, $573-585$.

(2) Dkhissi, A.; Adamowicz, L.; Maes, G. Density Functional Theory Study of the Hydrogen-Bonded Pyridine-H2O Complex: A Comparison with RHF and MP2 Methods and with Experimental Data. J. Phys. Chem. A 2000, 104, 2112-2119.

(3) Pápai, I.; Jancsó, G. Hydrogen Bonding in Methyl-Substituted Pyridine-Water Complexes: A Theoretical Study. J. Phys. Chem. A 2000, 104, 2132-2137.

(4) Wu, J.; Zhong, A.; Yan, H.; Dai, G.; Chen, H.; Liang, H. Study on the nature of interaction of pyrrole with various hydrides. Chem. Phys. 2011, 386, 45-49.

(5) Kumar, A.; Kołaski, M.; Kim, K. S. Ground state structures and excited state dynamics of pyrrole-water complexes: $\mathrm{Ab}$ initio excited state molecular dynamics simulations. J. Chem. Phys. 2008, 128, 034304.

(6) Sicilia, M. C.; Niño, A.; Muñoz-Caro, C. Mechanism of pyridine protonation in water clusters of increasing size. J. Phys. Chem. A 2005, 109, 8341-8347.

(7) Sicilia, M. C.; Muñoz-Caro, C.; Niño, A. Theoretical Analysis of Pyridine Protonation in Water Clusters of Increasing Size. ChemPhysChem 2005, 6, 139-147.

(8) Zborowski, K. K. Aromaticity of benzene in condensed phases. A case of a benzene-water system. Chem. Phys. Lett. 2014, 602, 1-3.

(9) Zborowski, K. K.; Szatyłowicz, H.; Krygowski, T. M. Solvent influence on intramolecular interactions and aromaticity in meta and para nitroanilines. Struct. Chem. 2020, 31, 1717-1728.

(10) Poater, J.; Solà, M.; Duran, M.; Fradera, X. Effects of solvation on the pairing of electrons in a series of simple molecules and in the Menshutkin reaction. J. Phys. Chem. A 2001, 105, 6249-6257.

(11) Poater, J.; Swart, M.; Guerra, C. F.; Bickelhaupt, F. M. Selectivity in DNA replication. Interplay of steric shape, hydrogen bonds, $\pi$ stacking and solvent effects. Chem. Commun. 2011, 47, 7326-7328. 
(12) Poater, J.; Swart, M.; Bickelhaupt, F. M.; Fonseca Guerra, C. BDNA structure and stability: the role of hydrogen bonding, $\pi$ - $\pi$ stacking interactions, twist-angle, and solvation. Org. Biomol. Chem. 2014, 12, $4691-4700$.

(13) El-Hamdi, M.; Tiznado, W.; Poater, J.; Solà, M. An Analysis of the Isomerization Energies of 1,2-/1,3-Diazacyclobutadiene, Pyrazole/ Imidazole, and Pyridazine/Pyrimidine with the Turn-Upside-Down Approach. J. Org. Chem. 2011, 76, 8913-8921.

(14) Curutchet, C.; Poater, J.; Solà, M.; Elguero, J. Analysis of the Effects of N-Substituents on Some Aspects of the Aromaticity of Imidazoles and Pyrazoles. J. Phys. Chem. A 2011, 115, 8571-8577.

(15) Frisch, M. J.; Trucks, G.; Schlegel, H. B.; et al. Gaussian 09, Revision A. 1; Gaussian Inc: Wallingford CT, 2009.

(16) Schmidt, M. W.; Baldridge, K. K.; Boatz, J. A.; Elbert, S. T.; Gordon, M. S.; Jensen, J. H.; Koseki, S.; Matsunaga, N.; Nguyen, K. A.; Su, S.; Windus, T. L.; Dupuis, M.; Montgomery, J. A. General atomic and molecular electronic structure system. J. Comput. Chem. 1993, 14, 1347-1363.

(17) Becke, A. D. Density-functional exchange-energy approximation with correct asymptotic behavior. Phys. Rev. A 1988, 38, 3098.

(18) Ditchfield, R.; Hehre, W. J.; Pople, J. A. Self-Consistent Molecular-Orbital Methods. IX. An Extended Gaussian-Type Basis for Molecular-Orbital Studies of Organic Molecules. J. Chem. Phys. 1971, 54, 724.

(19) Hariharan, P. C.; Pople, J. A. The influence of polarization functions on molecular orbital hydrogenation energies. Theor. Chim. Acta 1973, 28, 213.

(20) Tomasi, J.; Mennucci, B.; Cammi, R. Quantum mechanical continuum solvation models. Chem. Rev. 2005, 105, 2999-3094.

(21) Wolinski, K.; Hinton, J. F.; Pulay, P. Efficient implementation of the gauge-independent atomic orbital method for NMR chemical shift calculations. J. Am. Chem. Soc. 1990, 112, 8251-8260.

(22) Krygowski, T. M. Crystallographic studies of inter- and intramolecular interactions reflected in aromatic character of .pi-electron systems. J. Chem. Inf. Comput. Sci. 1993, 33, 70-78.

(23) Schleyer, P. v. R.; Maerker, C.; Dransfeld, A.; Jiao, H.; van Eikema Hommes, N. J. R. Nucleus-independent chemical shifts: A simple and efficient aromaticity probe. J. Am. Chem. Soc. 1996, 118, 6317-6318.

(24) Poater, J.; Fradera, X.; Duran, M.; Solà, M. The delocalization index as an electronic aromaticity criterion: Application to a series of planar polycyclic aromatic hydrocarbons. Chem.-Eur. J. 2003, 9, 400406.

(25) Matito, E.; Duran, M.; Solà, M. The aromatic fluctuation index (FLU): A new aromaticity index based on electron delocalization. J. Chem. Phys. 2005, 122, 014109.

(26) Giambiagi, M.; Segre de Giambiagi, M.; dos Santos Silva, C. D.; Paiva de Figueiredo, A. Multicenter bond indices as a measure of aromaticity. Phys. Chem. Chem. Phys. 2000, 2, 3381-3392.

(27) Bultinck, P.; Ponec, R.; van Damme, S. Multicenter bond indices as a new measure of aromaticity in polycyclic aromatic hydrocarbons. $J$. Phys. Org. Chem. 2005, 18, 706-718.

(28) Feixas, F.; Matito, E.; Poater, J.; Solà, M. Quantifying aromaticity with electron delocalisation measures. Chem. Soc. Rev. 2015, 44, 64346451.

(29) Feixas, F.; Matito, E.; Poater, J.; Solà, M. On the performance of some aromaticity indices: A critical assessment using a test set. $J$. Comput. Chem. 2008, 29, 1543-1554.

(30) Bader, R. F. W. Atoms in Molecules: A Quantum Theory; Halpen, J., Green, M. L. H., Eds.; The International Series of Monographs of Chemistry; Clarendon Press: Oxford, 1990.

(31) Keith, T. A. AIMAll, version 11.10.16; TK Gristmill Software: Overland Park KS, USA, 2011, www.aim.tkgristmill.com.

(32) Matito, E. ESI-3D: Electron Sharing Indexes Program for 3D Molecular Space Partitioning; Institute of Computational chemistry: Girona, 2014.

(33) Matito, E.; Feixas, F.; Solà, M. Electron delocalization and aromaticity measures within the Hückel molecular orbital method. J. Mol. Struct.: THEOCHEM 2007, 811, 3-11.
(34) Cioslowski, J. A new population analysis based on atomic polar tensors. J. Am. Chem. Soc. 1989, 111, 8333-8336.

(35) Bańkowski, K. Poradnik fizykochemiczny (in polish, Physicochemical Handbook); WNT: Warsaw, 1974.

(36) Poater, J.; Bofill, J. M.; Alemany, P.; Solà, M. Role of electron density and magnetic couplings on the nucleus-independent chemical shift (NICS) profiles of [2.2] paracyclophane and related species. J. Org. Chem. 2006, 71, 1700-1702.

(37) Cyrañski, M. K.; Krygowski, T. M.; Katritzky, A. R.; Schleyer, P. v. R. To What Extent Can Aromaticity Be Defined Uniquely? †. J. Org. Chem. 2002, 67, 1333-1338.

(38) Wu, J. I.; Jackson, J. E.; Schleyer, P. v. R. Reciprocal Hydrogen Bonding-Aromaticity Relationships. J. Am. Chem. Soc. 2014, 136, $13526-13529$. 